\title{
PERFIL DA CLIENTELA ATENDIDA PELO ENFERMEIRO NA ESTRATÉGIA ACOLHIMENTO MÃE-BEBÊ
}

\author{
Jessica Lima Silva ${ }^{1}$, Wânia Priscila Andrade de Melo ${ }^{1}$, Renata Abreu Sá ${ }^{1}$, Gláucia Cristina Lima da Silva ${ }^{1}$
} Nathália dos Santos Trindade ${ }^{1}$, Maria Helena Nascimento Souza ${ }^{2}$

\begin{abstract}
RESUMO: Trata-se de um estudo quantitativo do tipo descritivo-exploratório que objetivou identificar características sociodemográficas e de saúde de mães e bebês atendidos pelo enfermeiro na Estratégia Acolhimento Mãe-Bebê, em uma unidade básica de saúde do Município do Rio de Janeiro. Os dados foram coletados em 26 prontuários que possuíam o protocolo Roteiro de Atividades da Consulta do Acolhimento Mãe-Bebê, em 2011. A análise dados foi feita com o uso do software Epi-Info 3.5.2. Os resultados mostram que 61,3\% das mulheres estavam na faixa etária entre 20 a 30 anos; $46,2 \%$ haviam realizado parto normal; 7,7\% apresentavam alteração na cicatriz cirúrgica e $11,5 \%$ alteração nas mamas. Com relação aos recém-nascidos, $50 \%$ possuíam de 2 a 7 dias de vida; a maioria $(96,2 \%)$ encontrava-se em aleitamento materno exclusivo e 23,1\% apresentavam icterícia. Conclui-se que o conhecimento do perfil da clientela atendida no Acolhimento Mãe-Bebê, pelo enfermeiro, constitui importante subsídio para o desenvolvimento de medidas de promoção à saúde que visem redução da morbidade e da mortalidade materno-infantil.
\end{abstract}

DESCRITORES: Saúde materno-infantil; Enfermagem; Atenção primária à saúde.

\section{PROFILE OF THE CLIENTELE ATTENDED BY THE NURSE IN THE MOTHER-AND- BABY EMBRACEMENT STRATEGY}

\begin{abstract}
This quantitative study of the descriptive-exploratory type aimed to identify socio-demographic and health characteristics of mothers and babies attended by nurses in the Mother-and-Baby Embracement Strategy in a primary health care unit in the city of Rio de Janeiro. The data was collected from 26 hospital records which had the Guide for Motherand-Baby Embracement Consultation Activities protocol, in 2011. The data was analyzed using the Epi-infor 3.5.2 software. The results show that $61.3 \%$ of the women were in the age range 20 to 30 years old; $46.2 \%$ had had a normal birth; $7.7 \%$ had changes in the surgical scar and $11.5 \%$ had changes in their breasts. In relation to the new-borns, $50 \%$ were between 2 and 7 days old; the majority (96.2\%) were being exclusively breast-fed, and $23.1 \%$ presented jaundice. It is concluded that knowledge of the profile of the clientele attended in the Mother-and-Baby Embracement, by the nurse, constitutes important support for carrying out health promotion measures which aim to reduce maternal and infant morbidity and mortality. DESCRIPTORS: Maternal and infant health; Nursing; Primary health care.

\section{PERFIL DEL CLIENTE ATENDIDO POR EL ENFERMERO EN LA ESTRATEGIA ACOGIMIENTO MADRE BEBÉ}

RESUMEN: Este es un estudio cuantitativo del tipo descriptivo exploratorio que tuvo el objetivo de identificar características sociodemográficas y de salud de madres y bebés atendidos por el enfermero en la Estrategia Acogimiento Madre Bebé, en una unidad básica de salud del Municipio de Rio de Janeiro. Los datos fueron obtenidos en 26 prontuarios que poseían el protocolo Guion de Actividades de la Consulta del Acogimiento Madre Bebé, en 2011. El análisis de datos fue hecho con el uso del software Epi-Info 3.5.2. Los resultados muestran que 61,3\% de las mujeres estaban en la franja etaria entre 20 y 30 años; $46,2 \%$ habían realizado parto normal; 7,7\% presentaban alteración en la cicatriz quirúrgica y $11,5 \%$ alteración en las mamas. Acerca de los recién nacidos, $50 \%$ poseían de 2 a 7 días de vida; la mayoría $(96,2 \%)$ estaba en amamantamiento materno exclusivo y $23,1 \%$ presentaban ictericia. Se concluye que el conocimiento del perfil de la clientela atendida en el Acogimiento Madre Bebé, por el enfermero constituye importante subsidio para el desarrollo de medidas de promoción a la salud para la reducción de la mortalidad materno infantil.

DESCRIPTORES: Salud materno infantil; Enfermería; Atención primaria a la salud.

${ }^{1}$ Acadêmica do Curso de Graduação da Escola de Enfermagem Anna Nery da Universidade Federal do Rio de Janeiro - EEAN UFRJ. ${ }^{2}$ Enfermeira. Doutora em Enfermagem. Professora do Departamento de Enfermagem de Saúde Pública e Coletiva e do Programa de Pós Graduação da EEAN UFRJ. Membro do Grupo de Pesquisa de Enfermagem e Saúde. 


\section{INTRODUÇÃO}

A Estratégia Acolhimento Mãe-Bebê na Unidade Básica de Saúde, após alta da maternidade, foi implantada no Município do Rio de Janeiro em 2003, cumprindo as linhas de cuidado estabelecidas pela Agenda de Compromissos para a Saúde Integral da Criança e Redução da Mortalidade Infantil do Ministério da Saúde. Como uma das linhas de cuidado, a Agenda estabelece a Primeira Semana Saúde Integral em que todo recém-nascido (RN) deve ser acolhido na Unidade Básica de Saúde (UBS) durante a primeira semana de $\operatorname{vida}^{(1-2)}$.

Estudos demonstram que a atenção de profissionais de saúde à mãe e bebês nos primeiros meses de vida é de extrema importância para que possam ser estabelecidas as bases de uma boa saúde ${ }^{(3)}$. Em parceria com Programa de Saúde da Mulher, a Estratégia Acolhimento Mãe-Bebê tem por objetivos: estabelecer uma referência para uma recepção humanizada, após alta da maternidade, do binômio mãe-bebê na UBS mais próxima de sua residência; realizar as ações preconizadas para a $1^{\mathrm{a}}$ semana de vida do bebê e, no caso da mulher, as ações referentes à $1^{\mathrm{a}}$ semana após o parto; e possibilitar o estabelecimento precoce do vínculo da família com essa Unidade de Saúde ${ }^{(4)}$.

A decisão da mãe em procurar uma UBS é de grande importância para o primeiro contato das crianças com os serviços, além de ser necessária uma rede de referência, após a alta da maternidade, o que pode facilitar o início à assistência imediata da criança em uma unidade básica ${ }^{(5)}$.

$\mathrm{O}$ enfermeiro tem sido o profissional da equipe de saúde que realiza o acolhimento do binômio mãe e filho na primeira semana pós parto. E em sua prática assistencial é essencial o conhecimento da realidade da clientela atendida para melhor eficácia das ações desenvolvidas durante o atendimento ${ }^{(6)}$. Nota-se uma lacuna no conhecimento sobre as características de mães e recém nascidos que comparecem à UBS para a consulta na primeira semana pós- parto. Neste sentido, realizou-se esta investigação que teve como objetivo identificar características sociodemográficas e de saúde das mães e bebês atendidos pelo enfermeiro na Estratégia Acolhimento Mãe-Bebê, em uma unidade básica de saúde do município do Rio de Janeiro.

\section{MÉTODO}

Trata-se de um estudo documental, do tipo descritivo-exploratório, de abordagem quantitativa, realizado em uma UBS do Município do Rio de Janeiro, a partir da relação de prontuários de crianças atendidas pelas enfermeiras do setor de Puericultura, na consulta da Estratégia Acolhimento Mãe-Bebê, no mês de janeiro de 2011.

Para a definição da amostra considerou-se como critério de inclusão os prontuários que possuíam o protocolo denominado Roteiro de Atividades da Consulta do Acolhimento Mãe-Bebê, com data de preenchimento correspondente ao período de 02 a 31 de janeiro de 2011. Assim, a amostra foi constituída por 26 prontuários.

Do Roteiro da Estratégia Acolhimento Mãe-Bebê foram obtidas as seguintes informações: idade materna, tipo do parto, condições de saúde materna, idade do $\mathrm{RN}$, condições de saúde do $\mathrm{RN}$ no período pós-parto e tipo de alimentação do RN no momento do Acolhimento. Para o tratamento dos dados foi utilizado o software Epi-Info 3.5.2. Os resultados foram apresentados sob a forma de tabelas e analisados com auxílio de referencial teórico pertinente ao tema.

O presente estudo obteve a aprovação do Comitê de Ética e Pesquisa da Secretaria Municipal de Saúde do Rio de janeiro sob o número 129/08 e teve a autorização da direção da UBS.

\section{RESULTADOS}

Com relação às características das mulheres verificou-se que $15,4 \%$ delas eram menores de 19 anos e $61,3 \%$ possuíam idade entre 20 a 30 anos; $50 \%$ tiveram parto cesárea e $69,2 \%$ apresentaram cicatriz cirúrgica sem alterações. Quanto às condições de saúde na primeira semana pós parto, $11,5 \%$ das mulheres referiram febre, $15,4 \%$ apresentaram sangramento em excesso e $42,3 \%$ apresentaram algum tipo de alteração na mama, como fissura ou ingurgitamento mamário (Tabela 1).

De acordo com a tabela 2, verificou-se que $50 \%$ dos RN encontrava-se na primeira semana de vida. Com relação às intercorrências de saúde observou-se que a maioria não apresentou lesão de pele, icterícia ou alterações no coto umbilical. Quanto à alimentação, $96,2 \%$ dos RN estavam recebendo apenas o leite materno como alimento (Tabela 2). 
Tabela 1 - Distribuição das mulheres atendidas na consulta da Estratégia Acolhimento Mãe-Bebê. Rio de Janeiro, 2011

\begin{tabular}{lcc}
\hline Características & N (26) & \% \\
\hline Idade (em anos) & 04 & 15,4 \\
$<19$ & 05 & 19,1 \\
$20-25$ & 07 & 26,8 \\
$25-30$ & 10 & 38,3 \\
$>30$ & & \\
Tipo de parto & 13 & 50 \\
Cesárea & 12 & 46,2 \\
Normal & 01 & 3,8 \\
Fórcipe & & \\
Cicatriz cirúrgica & 18 & 69,2 \\
Sem alterações & 02 & 7,7 \\
Com alterações & 06 & 23,1 \\
Sem informações & & \\
Febre & 23 & 88,5 \\
Não & 03 & 11,5 \\
Sim & & \\
Sangramento normal & 04 & 15,4 \\
Não & 22 & 84,6 \\
Sim & & \\
Avaliação das mamas & 14 & 53,8 \\
Sem alterações & 11 & 42,3 \\
Com alterações & 01 & 3,9 \\
Sem informações & & \\
\hline
\end{tabular}

\section{DISCUSSÃO}

No presente estudo observou-se que a idade materna mais prevalente encontrava-se acima de 25 anos, o que corrobora com estudos que apontam para o fato da maternidade ser cada vez mais tardia, devido às mudanças no papel da mulher na sociedade, contribui para uma queda da fecundidade, maior uso de contraceptivos e adiamento da momento da gravidez ${ }^{(7-8)}$.

Em relação ao tipo de parto, conforme os resultados obtidos, houve maior prevalência do tipo cesárea do que o normal, esse valor pode ser associado às complicações na hora do parto, ao desejo da ligadura das trompas, pelo fato da mulher não querer sentir a dor durante o parto ou devido ao histórico de parto cesário anterior ${ }^{(8)}$.

Os cuidados e orientações prestados na primeira semana de saúde integral são fundamentais, tanto para a saúde da mãe como a do bebê. Pois, além de avaliar as condições de saúde da criança, é avaliado as condições de saúde da mãe, no período puerperal e, portanto, deve ser incentivado desde o pré-natal até a saída da maternidade ${ }^{(9)}$.
Tabela 2- Avaliação dos recém nascidos atendidos na consulta da Estratégia Acolhimento Mãe-Bebê. Rio de Janeiro, 2011

\begin{tabular}{lcc}
\hline Características & N (26) & \% \\
\hline Idade (em dias) & 13 & 50 \\
2 a 7 & 07 & 27 \\
8 a 15 & 06 & 23 \\
16 ou mais & & \\
Lesão de pele & 25 & 96,1 \\
Não & 01 & 3,8 \\
Sim & & \\
Icterícia & 20 & 76,9 \\
Não & 06 & 23,1 \\
Sim & & \\
Coto umbilical & 26 & 100 \\
Sem alterações & & \\
Tipo de alimentação & 25 & 96,1 \\
Somente leite materno & & 3,8 \\
Leite materno + & 01 & \\
leite artificial & & 53,8 \\
Avaliação das mamas & & 42,3 \\
Sem alterações & 14 & 3,9 \\
Com alterações & 11 & \\
Sem informações & 01 & \\
\hline
\end{tabular}

Os resultados mostraram que a consulta do enfermeiro na Estratégia Acolhimento Mãe-Bebê propicia que o enfermeiro esteja atento aos agravos que podem acometer a mãe no período pós parto, como a hemorragia, temperatura elevada ou alteração nas mamas. A presença de lóquios sanguíneos é considerada normal até o $5^{\circ}$ dia após o parto, semelhante à menstruação e de volume variável, com odor característico. Após o $5^{\circ}$ dia, torna-se serossanquíneo e após o $10^{\circ}$ dia, seroso. A presença de odor fétido pode indicar a presença de infecção. Cabe ressaltar que nesse período é formado um tamponamento dos vasos pela compreensão do miométrio para posteriormente formar trombos que vão impedir a perda de sangue ${ }^{(10)}$. No período de 24 horas após o parto, a mulher pode apresentar um estado febril $\left(36,8\right.$ a $\left.37,9^{\circ} \mathrm{C}\right)$ e não deve ser compreendido como um quadro infeccioso. No entanto, o examinador deve estar atento, pois também pode indicar um quadro infeccioso puerperal. A respeito das alterações da mama, as puérperas devem ser devidamente orientadas quanto à "pega" correta, evitando que o ato de amamentar seja incomodo e doloroso, corroborando 
para o desmame precoce. Nesse contexto, é fundamental a intervenção da Enfermagem por meio da educação em saúde a fim de minimizar esses $\operatorname{riscos}^{(6,11)}$.

Conforme os dados obtidos a maioria das crianças atendidas encontrava-se com boas condições de saúde nos primeiros dias de vida. Isto pode ser associado ao fato das mães, ao participarem do pré-natal e no alojamento conjunto, receberem boa orientação dos cuidados básicos com a criança, da limpeza e cuidado com o coto umbilical para não gerar nenhuma alteração, como uma infecção, da higienização do bebê, e da importância do aleitamento materno até os seis meses, sem necessidade de complementação ${ }^{(12)}$.

Embora a maioria das crianças apresentasse boas condições de saúde, foi constatado icterícia em 23,1\%. A hiperbilirrubemia é um achado encontrado em cerca de $63 \%$ dos $\mathrm{RN}$ nos primeiros dias de vida, e representa um período de adaptação do metabolismo à bilirrubina, não sendo necessário intervenção terapêutica ${ }^{(6)}$.

Com relação à alimentação das crianças atendidas, observou-se a predominância do aleitamento materno exclusivo, indo ao encontro das propostas do MS que, seguindo a recomendação da Organização Mundial da Saúde, indica a amamentação excluviva até os seis primeiros meses de vida ${ }^{(1-2,4)}$. A amamentação é um processo que estreita a relação entre a mãe e o bebê, além de ter implicações no estado nutricional da criança, no desenvolvimento do sistema imune, cognitivo e emocional, repercutindo também na saúde mental e física da mãe. O aleitamento materno é fundamental para a saúde da criança, dada a sua contribuição na diminuição da mortalidade infantil, das chances de desenvolver alergias, o risco de hipertensão, diabetes, obesidade, além de evitar diarréias, infecção respiratória entre outros. Para tanto, é necessário a aplicação de uma técnica correta para amamentação, evitando o aparecimento de lesões na mama ${ }^{(13)}$.

Nesta perspectiva verifica-se que a consulta do enfermeiro ao binômio mãe-filho no período pós parto mostra-se como uma ferramenta importante para a detecção precoce de agravos à saúde, realização de educação em saúde e para o acompahamento integral do crescimento e desenvolvimento da clientela atendida ${ }^{(6,14)}$.

Este trabalho abrangeu uma pequena parcela dos atendimentos realizados pelos enfermeiros em seu cotidiano assistencial, uma vez que foi restrito aos atendientos de um determinado mês.

\section{CONCLUSÃO}

Diante dos resultados obtidos nesta pesquisa constata-se que a maioria das mães e bebês atendidos no Acolhimento Mãe-Bebê estava em boa condição de saúde.No entanto, a ocorrência de problemas como alteração na cicatriz cirúrgica, nas mamas, febre, sangramento excessivo ou aleitamento misto, revelam a importância do desenvolvimento de ações de promoção à saúde e prevenção de agravos, mediante consulta ao binômio mãe-filho, desde a primeira semana pós parto.

Conclui-se que o conhecimento do perfil da clientela atendida no Acolhimento Mãe-Bebê, pelo enfermeiro, constitui em um importante subsídio para o desenvolvimento de medidas de promoção à saúde, que visem a redução da morbidade e da mortalidade infantil.

\section{AGRADECIMENTOS}

Agradecemos a Jessica Ribeiro de Pinho e a Natasha Viegas Macedo pela colaboração nessa pesquisa.

\section{REFERÊNCIAS}

1. Secretaria Municipal de Saúde (RJ). Acolhimento mãebebê na unidade básica após a alta da maternidade. Rio de Janeiro: Gerência de Programas de Saúde da Criança; 2003.

2. Ministério da Saúde (BR). Secretaria de Atenção à Saúde. Departamento de Ações Programáticas Estratégicas. Agenda de compromissos para a saúde integral da criança e redução da mortalidade infantil. Brasília: Ministério da Saúde; 2004.

3. Bueno LGS, Keiko TM. Aconselhamento em amamentação e sua prática. J. Pediatr. [Internet] 2004;80(5) [acesso em 14 nov 2011]. Disponível: http:// dx.doi.org/10.1590/S0021-75572004000700003

4. Secretaria Municipal de Saúde (RJ). Linha de cuidado da atenção integral à saúde da criança. Rio de Janeiro: Gerência de Programas de Saúde da Criança; 2010.

5. Souza IS. Avaliação do fluxo de crianças entre a alta da maternidade e o atendimento na unidade básica de saúde do SUS, no Município do Rio de Janeiro [dissertação]. Rio de Janeiro (RJ): Escola Nacional de Saúde Pública; 2007.

6. Souza MHN, Gomes TNC, Paz EPA, Trindade CS, Veras RCC. Estratégia acolhimento mãe-bebê: aspectos relacionados à clientela atendida em uma unidade básica de saúde do município do Rio de Janeiro. Anna Nery. [Internet] 2011;15(4) [acesso em 14 nov 2011]. Disponível: http://dx.doi.org/10.1590/S141481452011000400003 
7. Borlot AMM, Trindade ZA. As tecnologias de reprodução assistida e as representações sociais de filho biológico. Universidade Federal do Espírito Santo. Estud. Psicol. [Internet] 2004;9(1) [acesso em 16 nov 2011]. Disponível: http://dx.doi.org/10.1590/S1413294X2004000100008

8. Dias MAB, Domingues RMSM, Pereira APE, Fonseca SC, Gama SGN, Filha MMT, et al. Trajetória das mulheres na definição pelo parto Cesário: estudo de caso em duas unidades do sistema de saúde suplementar do estado do Rio de Janeiro. Ciênc. saude colet. [Internet] 2008;13(5) [acesso em 14 nov 2011]. Disponível: http:// dx.doi.org/10.1590/S1413-81232008000500017

9. Ministério da Saúde (BR). Pré-natal e Puerpério: atenção qualificada e humanizada. Ministério da Saúde, Secretaria de Atenção à Saúde, Departamento de Ações Programáticas Estratégicas. Brasília: Ministério da Saúde; 2005.

10. Ministério da Saúde (BR). Parto, aborto e puerpério: assistência humanizada à mulher. Ministério da Saúde, Secretaria de Políticas de Saúde, Área Técnica da Mulher. Brasília: Ministério da Saúde; 2001.

11. Ravelli APX. Consulta puerperal de Enfermagem: uma realidade na Ponta Grossa, Paraná, Brasil. Rev. Gaúcha Enferm. [Internet] 2008; 29(1) [acesso em 16 nov 2011]. Disponível: http://seer.ufrgs.br/ RevistaGauchadeEnfermagem/article/view/5264/2998.

12. Fonseca LMM, Scochi CGS, Mello DF. Educação em saúde de puérperas em alojamento neonatal: aquisição de conhecimento mediato pelo uso de um jogo educativo. Rev. Latino-Am Enfermagem. [Internet] 2002;10(2) [acesso em 16 nov 2011]. Disponível: http:// dx.doi.org/10.1590/S0104-11692002000200007

13. Ministério da Saúde (BR). Saúde da criança: nutrição infantil, aleitamento materno e alimentação complementar. Ministério da Saúde. Secretaria de Atenção à Saúde. Departamento de Atenção Básica. Brasília: Ministério da Saúde; 2009.

14. Vieira V, Fernandes C, Demitto M, Bercini L, Scochi $\mathrm{M}$, Marcon S. Puericultura na atenção primária à saúde: atuação do enfermeiro. Cogitare enferm. 2012;17(2):11925 . 